\title{
VIRTUAL FACE TO FACE MEETING FOR NON-TECHNICAL PERSONNEL
}

\author{
Lukas Tanutama ${ }^{1}$, Santoso Budijono ${ }^{2}$
}

\author{
Management Department, BINUS Business School Undergraduate Program, \\ Bina Nusantara University \\ Jl. Kebon Jeruk Raya No. 27, Kebon Jeruk, Jakarta 11530, Indonesia \\ ${ }^{1}$ lukast12@binus.edu, ${ }^{2}$ santoso_budijono@binus.ac.id
}

Received: January $21^{\text {st }}, 2020 /$ Revised: February $12^{\text {th }} 2020 /$ Accepted: February $24^{\text {th }}, 2020$

\begin{abstract}
Covid-19 has impacted profoundly the business environment. Information and Communication Technology (ICT) becomes not only the major platform but also sustains the existence and running most business. Retail business becomes more dependent on technology. Small Business Enterprises (SME) have to jump and become savvy in ICT. The SME supply chain and marketing environment are moving toward ICT based platforms. On the other side the penetration of social media tool especially smartphone is widely spread. It can be safely assumed that every job holder whether entrepreneur, employee, or professionals or craftspeople is familiar with smartphones. The gap that must be bridged is using smartphone for business purposes. One of the business requirements that are not easy to replace is face to face communications. As Internet is ubiquitous and smartphones can easily access the Internet, there are numerous application that can resides both in computers and smartphones. The application that is closely similar to face to face meeting is known as Video Conference call. With this application it is possible to perform business briefing any time and anywhere provided there is Internet access. As business communication is not limited to technically savvy parties, a supportive system must be selected and introduced to them. We select Zoom as the application and develop a simple training syllabus. The syllabus has been implemented to a retail chain.
\end{abstract}

Keywords: smartphone, ICT, video conference, Zoom

\section{Introduction}

Covid-19 pandemic has impacted profoundly the business environment. Information and Communication Technology (ITC) becomes not only the major platform but also sustains the existence and running most business (ILO, 2020). The impact of COVID-19 on small businesses were surveyed at the first wave of the pandemic (Rahman, 2020). The survey was conducted in a developed country. It was found that there were several several happenings emerged. Most conspicuous are mass layoffs and closures occurred just a few weeks into the crisis.

Businesses weighed closures associated with the expected length of the crisis. Businesses also had widely varying prognosis about the probable duration of disruptions due to COVID (ILO, 2020). Even for small businesses in a developed country they are financially fragile: They temporarily closed largely due to decrease in demand and government regulation for public health concern (Lutfi, 2020). They have to reduce their active employment considerably. The impacts also varied across industries. Most impacted are retail, arts, entertainment including tour and travel and hospitality businesses. The finance, professional services, and real estate related businesses are less disrupted. These industries are able to move to technology-based activities such as remote works.

Retail businesses become more dependent on technology. Small Business Enterprises (SME) have to jump and become savvy in ITC. The SME supply chain and marketing environment are moving toward ITC based platforms (ILO, 2020).

As the government has implemented countermeasures to decrease Covid-19 spread such as social distancing, mask wearing and work-fromhome policies, many retail establishments must close, reduce capacity or reduce operating hours. To sustain their business, many migrated to online activities. The one major sector that have significantly been impacted are retailers. Organisations are shifting to online delivery and this is accelerating Indonesia's push towards a digital economy faster than originally planned. To 
capitalise on this, Indonesia needs to focus on strengthening its digital ecosystems by upgrading its information and communications technology (ICT) infrastructure, upskilling its ICT and digital skills workforce, and strengthening its ICT-related policies (ILO, 2020).

Economic impact of COVID-19 to Indonesia according to the Ministry of Manpower is rise of unemployment. Since the COVID-19 outbreak began, more than 2 million Indonesian workers have lost their jobs. This could certainly lead to increased poverty. In order to support businesses, workers and families that suffered from the economic disruption, the government announced an economic stimulus package. Research suggests that changes in attitudes and behaviour is leading to a new business environment. Consumers are staying home more forcing businesses to adapt to stay-at-home economy. Sectors are businesses related to health, online education, e-commerce are booming. This is a sign of the uptake of digital business and digital services. The digital transformation is naturally being pushed much earlier than initially planned. The transformation is almost immediate. E-commerce and digital business during COVID-19 takes off as consumers is forced to practice social distancing.

E-commerce companies in Indonesia are reporting higher volumes of online sales. Consumers increasingly turning to online channels to make purchases as the COVID-19 case count rose. People are buying health care and work-fromhome products, and groceries. The growth of online grocery shopping is assumed due to supermarkets reducing operating hours and limited accessibility of traditional market. The spike in transactions along with growth in new users ia accompanied by the shifts in the SME business model and consumption behaviour as Indonesians adjust to spending more time at home. The need for products which due to the social distancing imposed by the government can only be fulfilled effectively through online shopping. Consumers who have never purchased online are now embracing the digital marketplaces to acquire essential products. As the demand for online shopping is increasing, consumers need more technical skill to be able to perform their transactions.

COVID-19 has forced Indonesians to transform workplaces in order to continue operating. Companies that are able to utilize technology effectively and rethink their business models by integrating digital tools will have advantages over their competition. The pandemic has led to the need of increasing or at least introducing ICT skill of the common work force in order to perform in the new work environment. The increased ICT adoption positively impacts on the digital environment that is essential for digital economy.

To many business owners in Indonesia, the COVID-19 pandemic force them to digitally transform their businesses. Their working processes is geared to allow them to accelerate the changes. Businesses that do not innovate and operate capatalizing technology most likely will perish. Meanwhile, tech companies that provide software-based services are continuously launching products and services that could streamline or even replace the previous business process. Business owners should seize the opportunity to establish digital business models, strategies and collaboration agreements for its sustainability in the long run.

Through this crisis, we have seen that it is very risky for societies to ignore technological advancements in the face of evolving business models and general lifestyle trends. As the main policymaker, the government needs to provide the necessary digital infrastructures and robust digital policies to accelerate the digital transformation of Indonesia after the pandemic is over.

On the other side the penetration of social media tool especially smartphone is widely spread. It can be safely assumed that every job holder whether entrepreneur, employee, or professionals or craftspeople is familiar with smartphones. The gap that must be bridged is using smartphone for business purposes. One of the business requirements that are not easy to replace is face to face communications. As Internet is ubiquitous and smartphones can easily access the Internet, there are numerous application that can resides both in computers and smartphones. The application that is closely similar to face to face meeting is known as Video Conference call. With this application it is possible to perform business briefing any time and anywhere provided there is Internet access (Haman, 2020).

While the COVID-19 pandemic has accelerated people's adoption of e-commerce and online shopping platforms, poor digital literacy hamper efforts traditional market merchants across Indonesia go digital with their businesses. Most of the traders at traditional markets still view their trade in a very conventional way that must involve face-to-face transactions. 
The number of small and medium enterprises (SMEs) using online platforms for their business has risen and resulted in speeding up digitalization. SMEs that successfully adapt to the new consumer habit and continously innovate can expect surviving this crisis. The rise in online commerce among small businesses has taken place as many consumers avoid visiting shopping centers and move to online shopping platforms to comply with the social-distancing orders during the coronavirus pandemic. We note that consumers are faster in adapting to online than ever before. When consumers move online, this gives an opening to the merchants to tap new customers.

In concerning digital transformation, organizations consider the company culture presents one hurdle to their success. To ensure digital transformation is a sustainable success, training in skills to embrace new methodologies, mindsets and processes is absolutely essential. To develop a culture for full and successful digital transformation, company-wide transparency, communication, collaboration and development is essential. As the company's staff determines the sucessful shift toward digital transformation, it is necessary the business began to implement a host of tools that enable collaboration, innovation, and foster an entrepreneurial spirit based on ICT. Shift toward agile working and regular crossdepartmental meetings will give the business a sustainable business.

The pace of change related to the digital world is rapid and not expected to slow down anytime soon. To hurtle towards continual digital enlightenment at breakneck speed, employees across departments need access to resources and platforms that will empower them to constantly top up their skills and learn new proficiencies that will allow them to remain relevant and up to date in an incredibly competitive landscape.

To remain competitive in a digital environment, organizations must foster a culture of knowledge, sharing and continuous learning. Business leaders feel they need to equip their employees with skills to support business process adaption. Offering training or workshop to platforms, technologies and curriculums will allow them to continually upskill according to emerging trends and technological evolutions

The pace of change related to the digital world is not expected to slow down anytime soon. Employees across departments need access to resources and platforms that will empower them to constantly top up their skills and learn new proficiencies that will allow them to remain relevant and up to date in an incredibly competitive landscape (Das,2020).

The output target is remain competitive in a digital environment. The organizations must offer a culture of knowledge, sharing and continuous learning to all employees. The employees are access to platforms, technologies and curriculums that will allow them to continually upskill according to emerging trends and technological evolutions, Non technical employees especially front-liners and supporting and administrative staff should be familiar with the necessary technology. The initial step is enacling face to face real time virtual meeting or briefing. Preferably the application is residing in the employee's smartphone. They should be able to initiate a meeting besides participating in a meeting. The application could complement on line transaction due to the possibility of personal or limited party meeting similar to the traditional face to face meeting. (Ball, 2020)

\section{Implementation and Methods}

In retail business face to face communication such as daily briefing, end of day reporting, immediate problem solving is the normal practice. Quality of service depends on the agility of the people in charge solving unexpected situations. Before Covid-19 face to face communication is less of a problem because there are no restrictions. The main obstacle in the big cities are traffic and parking spot. The other obstacle is the number of face to face communication participants at one session. Each different location generally need additional arrangement. Normal telephone service or cellular telephone call is not feasible due to limited simultaneous participants and prohibitive cost. Application such as WA could be an alternative as most participants are familiar, but the limited number of participants and the difficulty in joining a session is an obstacle.

Video Conferencing application is the chosen application for virtual face to face communication. It complies to the requirements of common business practice of face to face business communication in terms of number of participants, number of locations, and cost. A minimum training is needed for certain persons such as supervisor or employees that urgently need to communicate to the company. The system supports visual communication as well and provides archiving of the meeting. The Covid-19 challenges could solved 
by the system selected and additional advantage compared to the traditional face to face meeting is time saving in terms of traveling time and location independence (Cytowic, 2020). As business communication is not limited to technically savvy personnel, the system should be user friendly, simple that the participants need only minimum training to use it (Dorset, 2020).

Finally an application called Zoom is selected. Zoom is used to support the Standard Operating Procedure of a Food and Beverages retail business that will be implement the solution. The employees that need regularly face to face communication meeting will follow the designed training syllabus. At the end of the training they will be able to do the virtual face to face communication either as a participant or as initiator. The syllabus designed for small business enterprises and in this case retail food and beverage chain. The training is based on direct hands-on activities. Each participant must be able to initiate a virtual face to face communication meeting. In this training the activities is based on smartphone as all participants are familiar and use it daily.

In this training there were 14 participants that are residing at various locations. Initially to enable the use of Zoom, they had to install the application software. Traditional communication was used to do the initial installation and the continued with running application. Each of the participants played the required role as demanded in virtual face to face communication until they are completely familiar and can be independent.

The hands-on training is started with personal Voice or Whatsapp instruction on installing Zoom application. Each participant is assumed have no experience or have never use Zoom application and instructed personally to take the four steps.

First step is create group, each group consist of 4 participant and one assistant trainer. This step is complex because participant not have experience for use Zoom application. All participant not familiar of using Application credential (username and password for application) and Email credential (username and password for email), and first contact and communication using Whatsapp.

Second step, the material for install and run Zoom application on smartphone will send to all participant via Whatsapp, to run this training must assist with 5 trainer, one act as master trainer and present how to install and setup Zoom application in each group and 4 other trainer will conduct practice of install and setup Zoom using WhatsApp communication.

Third step, after first trainer present the step of install and setup Zoom application at each group, other trainer will conduct step of install and run Zoom Application in each group that consist of 4 participant in one hour. The step of install and run Zoom application is (1) Setup email account if participant does not have it, (2) Install Zoom application from google play at participant smartphone, (3) Register Zoom using Gmail participant username (pay attention to username and password), (4) Open Gmail to activate Zoom register and finally trainer create meeting at Zoom and send link meeting using WhatsApp group to make all participant can join meeting using those link. Training process follows the following slides for the instructor: 


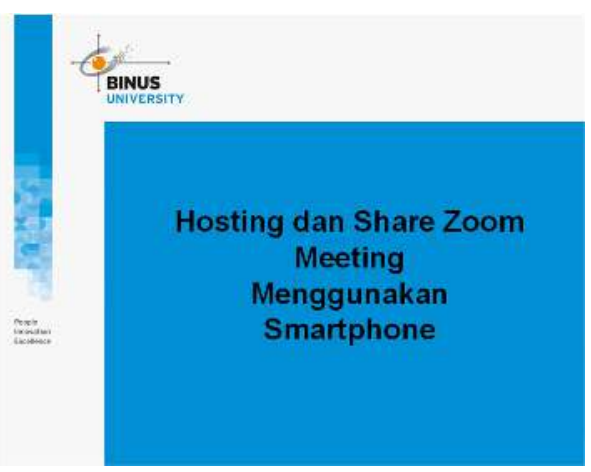

Figure 01 Slide 01

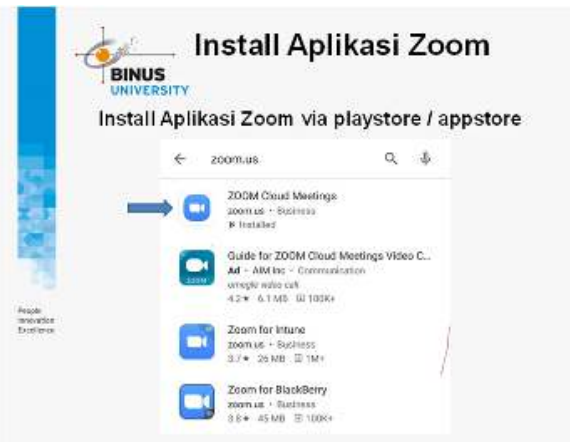

Figure 03 Slide 03

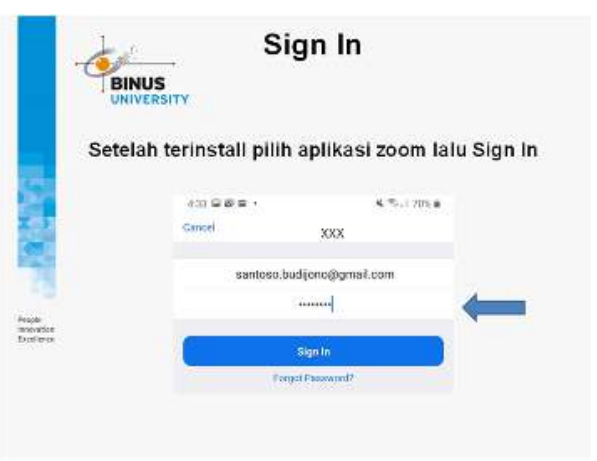

Figure 05 Slide 05

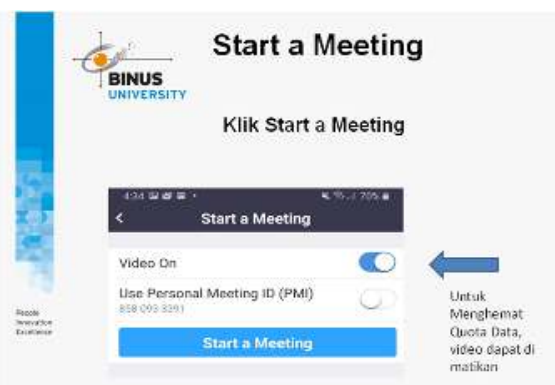

Figure 07 Slide 07

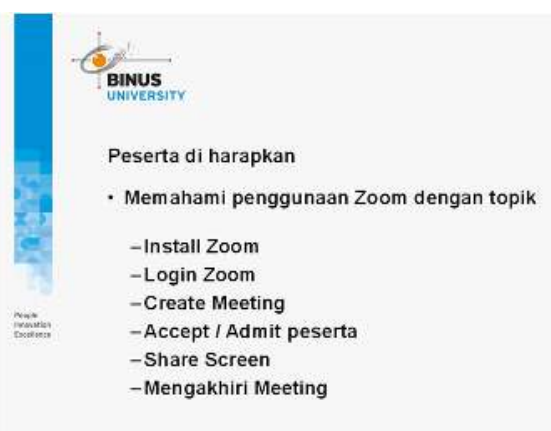

Figure 02 Slide 02

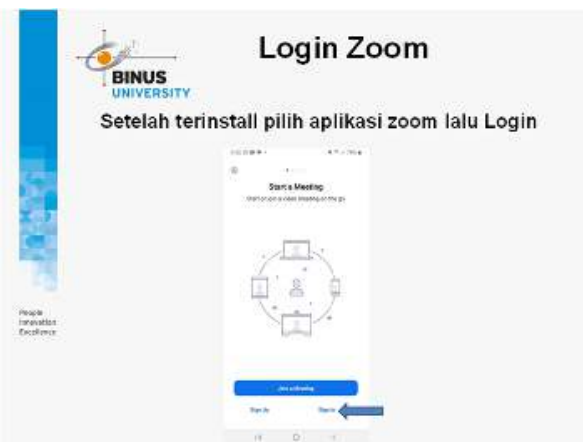

Figure 04 Slide 04

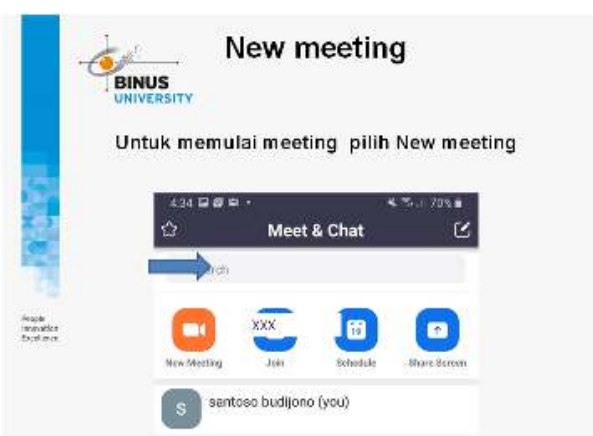

Figure 06 Slide 06

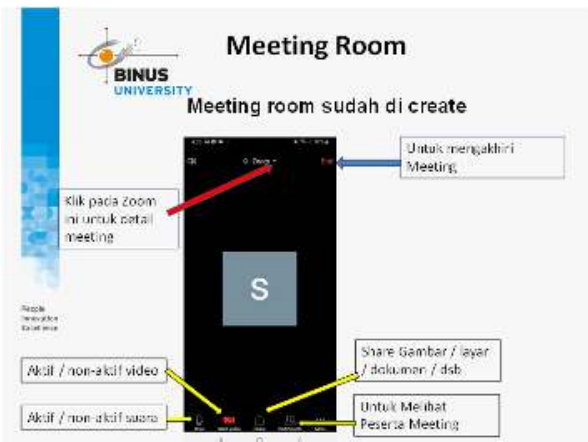

Figure 08 Slide 08 


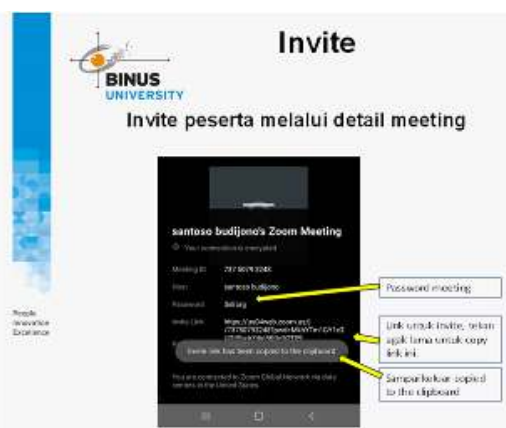

Figure 09 Slide 09

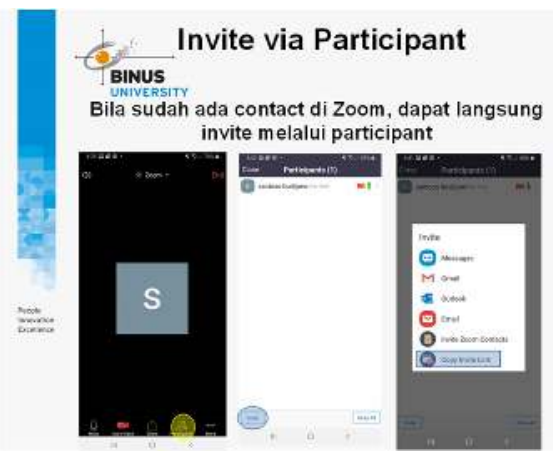

Figure 11 Slide 11

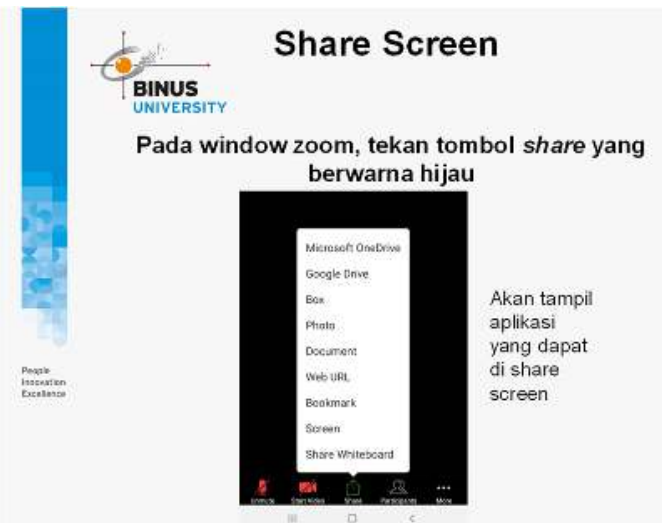

Figure 13 Slide 13

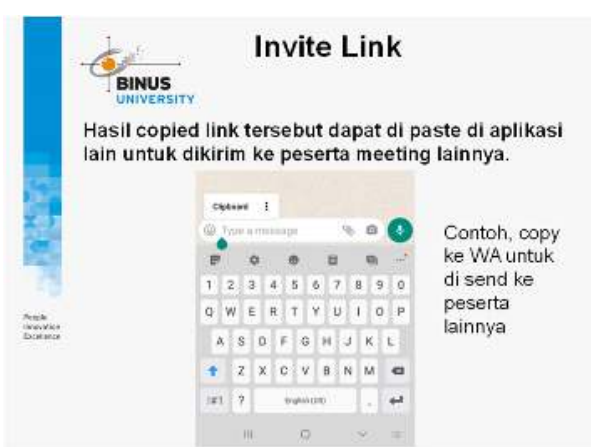

Figure 10 Slide 10

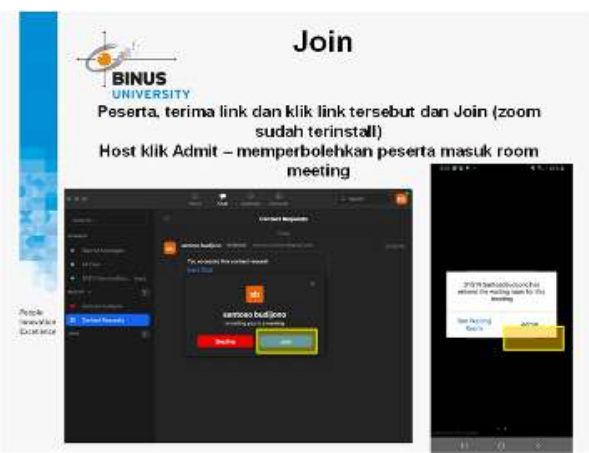

Figure 12 Slide 12

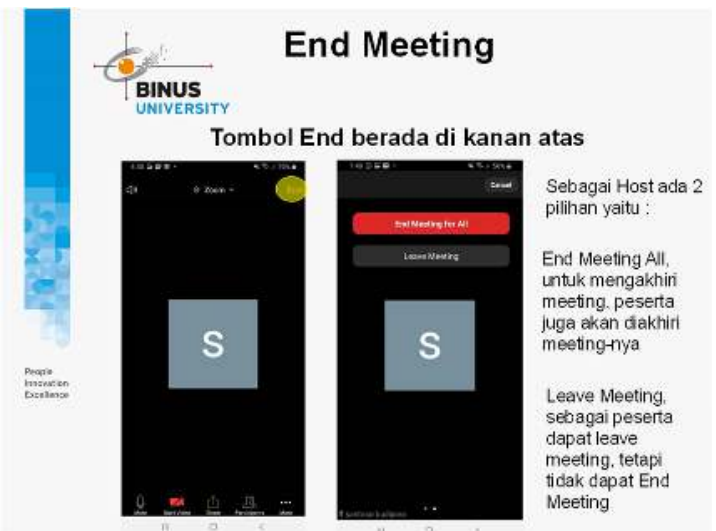

Figure 14 Slide 14 


\section{Result and Discussions}

The result of the training method as implemented resulted the great acceptance of the participants. They can now have face to face communication with their peers, supervisor or management for problem solving of obstacles that need resolution. They can now initiate a short meetings with short notice and invite the relevant parties. As this is a virtual face to face meeting and Internet is the infrastructure, geographical location and time present minimum obstacle. Moreover; smartphone is the main enabler for the participants. All participants is familiar on how to operate a smartphone and it is assumed that they are familiar with handling social media. The hands-on training with Zoom added a necessary digital business skill for the non-technical job holder. The retail business itself benefited from the skill acquired from the training for their operations. Problems and immediate actions can be easily executed as the relevant parties can provide their commitments. Additional benefit is the possibility of archiving the virtual face to face meeting which is not an easy matter in a conventional face to face meeting.

After these training, all participant have familiar with Zoom application, make Company HeadQuarter easily conduct virtual meeting at any time. Actualy these virtual meeting can reduce cost of traveling cost, cause crew at other city can attend meeting without have to come to company Head Office.

\section{Conclusions}

The hands-on taining with Zoom added a necessary digital business skill for the nontechnical job holder. The hands-on training for each participant enhanced the skill retention as shown from their actions in performing their exercise. The training can be replicated to other entities that need face to face meeting albeit virtual face to face and practically no special technical skill needed. Everybody that has a smartphone and regularly use it for social media or gaming activities could initiate and participate in a virtual face to face communications.

These Zoom virtual meeting will use participant's data quota. If a company allocate subsidy to its employees, attention must be paid to data usage and the costs. The employee must be able to provide usage reports in the zoom application.

\section{References}

Rahman, D.F. (2020). Most small businesses could shut down in six months due to COVID-19: UNIDO. The Jakarta Post. Retrieved from https://www.thejakartapost.com/news/2020/10/01/ most-small-businesses-could-shut-down-in-sixmonths-due-to-covid-19-unido.html

ILO-International Labour Organization. (2020). The clock is ticking for survival of Indonesian enterprises, jobs at risk-Key findings of the ILO SCORE Indonesia COVID-19 enterprise survey. Research Brief, Retrieved from https://www.ilo.org/wcmsp5/groups/public/--asia/---ro-bangkok/---ilojakarta/documents/publication/wcms_745055.pdf

Lutfi M., Buntuang P.C.D., Kornelius Y., Erdiyansyah and Hasanuddin B. (2020). The impact of social distancing policy on small and medium-sized enterprises (SMEs) in Indonesia. Problems and Perspectives in Management, 18(3), 492-503.

ILO-International Labour Organization. (2020). Staying competitive during COVID-19 with digital skills Retrieved from https://www.ilo.org/jakarta/info/public/pr/WCMS _745068/lang--en/index.htm

Haman K. (2020). Mastering the Virtual Practice: Virtual vs. Face-to-Face Meetings. Retrieved from https://www.alliancebernstein.com/library/Masteri ng-the-Virtual-Practice-Virtual-vs-Face-to-FaceMeetings.htm

Ball C. (2020). Face-to-Face Vs. Virtual Meetings: Which is Better? Retrieved from https://www.corbinball.com/article/44-virtualmeetings-web-meetings-video-andcollaboration/273-f2f-vs-virtual

Cytowic R.E. (2020), Can Apps Like Zoom Replace Face-to-Face Interaction? Retrieved from https://www.psychologytoday.com/intl/blog/thefallible-mind/202004/can-apps-zoom-replaceface-face-interaction

Dolan L. (2020). Zoom best practices for your small business. Retrieved from https://keap.com/business-success-blog/digitalmarketing/zoom-best-practices-for-your-smallbusiness 
Dorset, (2020).Meetings using Skype or Zoom in the COVID-19 lockdown. Dorset Chamber.News. Retrieved from https://dorsetchamber.co.uk/meetings-usingskype-or-zoom-in-the-covid-19-lockdown/

Das K, Gryseels M, Sudhir P, Tan K.T. (2020). Unlocking Indonesia's digital opportunity. Retrieved from Retrieved from https://www.mckinsey.com/ /media/McKinsey/L ocations/Asia/Indonesia/Our\%20Insights/Unlocki ng\%20Indonesias\%20digital\%20opportunity/Unl ocking_Indonesias_digital_opportunity.ashx 